\section{Gender differences in depression}

\author{
Critical review
}

MARCO PICCINELLI and GREG WILKINSON

\begin{abstract}
Background With few exceptions, the prevalence, incidence and morbidity risk of depressive disorders are higher in females than in males, beginning at midpuberty and persisting through adult life.
\end{abstract}

Aims To review putative risk factors leading to gender differences in depressive disorders.

Method Acritical review of the literature, dealing separately with artefactual and genuine determinants of gender differences in depressive disorders.

Results Although artefactual determinants may enhance a female preponderance to some extent, gender differences in depressive disorders are genuine. At present, adverse experiences in childhood, depression and anxiety disorders in childhood and adolescence, sociocultural roles with related adverse experiences, and psychological attributes related to vulnerability to life events and coping skills are likely to be involved. Genetic and biological factors and poor social support, however, have few or no effects in the emergence of gender differences.

\section{Conclusions Determinants of gender differences in depressive disorders are far from being established and their combination into integrated aetiological models continues to be lacking.}

\section{Declaration of interest No funding.} G.W. is Editor of the British Journal of Psychiatry.
Epidemiological findings point to a female preponderance in prevalence, incidence and morbidity risk of depressive disorders. Increased risk to females varies by diagnostic subtypes and is substantial for major depression, dysthymia, atypical depression and seasonal winter depression, although it does not occur in bipolar disorder (but females predominate in rapid-cycling and mixed-state episodes). Moreover, age is a crucial factor, and higher rates of depression in females are detected at mid-puberty through adult life, as opposed to a male preponderance until early adolescence. Finally, the female preponderance is not ubiquitous and is limited, or even absent, in traditional societies and in socially homogeneous samples (Piccinelli \& Gomez Homen, 1997). This paper aims to review putative risk factors leading to gender differences in depressive disorders. For this purpose, artefactual and genuine determinants will be considered separately (Weissman \& Klerman, 1977).

\section{ARTEFACTUAL \\ DETERMINANTS}

\section{Thresholds for caseness}

At present, the definitions of depressive disorders remain arbitrary because of the absence of clear markers or 'natural' thresholds in symptom distribution. The tendency of females to report more depressive symptoms than males might be responsible for their greater likelihood of meeting criteria for a depressive disorder, even though males and females with depressed mood report similar levels of subjective, social and occupational impairment (Angst \& Dobler-Mikola, 1984). Several studies have shown that a female preponderance in rates of depression occurs at low symptom thresholds and becomes more pronounced as the number of symptoms increases. Moreover, similar relationships between levels of depression and occupational impairment have been reported in males and females (Kessler et al, 1993; Fennig et al, 1994).

\section{Measurement procedures}

Clinical manifestations of depression differ by gender, with females more often reporting disturbances of appetite and sleep, fatigue, somatic anxiety and hypochondriasis (Frank et al, 1988; Young et al, 1990; Silverstein, 1999). Thus, the inclusion of gender-dimorphic items in rating scales and diagnostic algorithms may influence depression rates. In fact, gender differences in symptom profile tend to be limited in type and severity, and genderspecific response patterns on rating scales for depression do not seem fully to account for gender differences in levels of depression (Steer et al, 1989; Stommel et al, 1993).

\section{Effect of recall}

The issue of temporal stability in reporting mental disorders has received direct attention in several studies, suggesting that passage of time greatly affects accuracy of recall (Bromet et al, 1986; Dohrenwend, 1989). No consistent gender-specific recall patterns have been reported, which might result in an artefactual female preponderance in depression rates. Indeed, females outnumber males even when depressive episodes are assessed over short time periods preceding examination in order to limit the recall bias. Also, there are no significant gender differences in the temporal distribution of depressive episodes, no gender-by-time interaction in the likelihood of reporting depressive symptoms or, even, a better recall of past episodes in males (Coryell et al, 1992; Fennig et al, 1994). A sophisticated approach based on longitudinal design and corroborative witness reports, allowing for partial correction of the tendency of males to forget or minimise previous episodes, found a trend for higher lifetime rates of depression in females at the time of the emergence of gender differences in social roles (Wilhelm \& Parker, 1994; Wilhelm et al, 1997).

\section{Course of illness}

Gender differences in the course of depression, rather than in its occurrence, 
might be responsible for the higher prevalence rates among females. Findings are controversial: some studies report higher rates of first-onset depression in females rather than a greater number, or longer duration, of episodes (Kessler et al, 1993; Wilhelm et al, 1997); others show a female preponderance in recurrent and chronic depression (Stefànsson et al, 1994; Bracke, 1998). In any case, the course of illness loses its relevance in computing lifetime prevalence rates, which are consistently higher in females than in males.

Differential mortality in males and females with depression has been also considered but can hardly account for gender differences in depression, since these appear at puberty through adult life and decrease in older cohorts, when the differential effect of mortality is expected to be more pronounced.

\section{Help-seeking and illness behaviour}

Females are more likely to report physical and psychological symptoms and to seek medical help, although few gender differences have been detected in illness behaviour, sick role or defence style (Spinhoven \& Kooiman, 1997; Gijsbers van Wijk et al, 1999). Females do not appear to report milder symptoms than males on mental health scales (Tousignant et al, 1987) and gender does not seem to predict underreporting of psychological symptoms (Lyness et al, 1995; Cantwell et al, 1997).

\section{Depression spectrum}

Developmental pathways towards depression may differ by gender, with females suffering from pre-existing anxiety disorders and males experiencing more externalising disorders, such as alcoholism, antisocial personality and drug misuse. In this regard, the concept of 'depression spectrum disease' has been suggested to identify a specific gene-environment interaction leading to depression in females and alcoholism in males. At present, the evidence suggests that the above-mentioned disorders may share genetic and environmental factors with depression but cannot be considered simply as indirect manifestations of depression (Cadoret et al, 1996; Kendler et al, 1997; Stallings et al, 1997; Dawson \& Grant, 1998).

\section{EXPLANATORY FACTORS}

Several risk factors have been investigated. They are listed in Table 1.

\section{Familial environment and adverse experiences in childhood}

Genetic studies have shown that environmental factors shared in families do not substantially influence liability to major depression and do not contribute to observed gender differences (Kendler et al, 1995; McGuffin et al, 1996). None the less, the role of familial environment cannot be totally dismissed, because events occurring within families and not shared by family members remain candidates. Familial factors may contribute to vulnerability to depression in terms of personal attributes modulating the response to life events, with no increased risk of illness in the absence of such crises. Age and strictness of diagnostic criteria may be critical variables, since depressive illness in children, as well as milder forms of depression, seems to be largely related to unique or shared environmental factors (Brown, 1996;

Table I Risk factors explaining gender differences in depression

Childhood familial environment and adverse experiences

Prior depression and anxiety disorders

Social roles and cultural norms

Adverse life events

Vulnerability and coping style

Social support

Genetic factors

Gonadal hormones

Adrenal axis and thyroid axis

Neurotransmitter systems
Farmer, 1996; Harrington, 1996; Silberg et al, 1999).

Much attention has been devoted to parental separation/divorce (with resulting lack of child care in early years) and to the effects of physical and sexual abuse in childhood as possible risk factors for depression in adult life. Adverse experiences in childhood have been shown to increase the risk of later depression through several pathways, including: biological mechanisms (that is, long-term dysregulation of the hypothalamic-pituitary-adrenal axis); personal vulnerability (namely low self-esteem, helplessness, external locus of control, poor coping strategies); adverse environmental factors (such as lack of social support, low social status, ongoing difficulties like single parenthood or unplanned pregnancy); and a depression episode in teenage years (Kendler et al, 1993; Bifulco et al, 1998; Weiss et al, 1999). However, gender differences in the association between adverse experiences in childhood and adult depression have been poorly investigated. The available evidence suggests that early traumatic experiences may be partly responsible for a female 
preponderance in depression rates, since females are at greater risk of certain events (such as sexual abuse) and seem to be more sensitive to their depressogenic effects (Rodgers, 1994; Veijola et al, 1998).

\section{Prior depression and anxiety disorders}

Females are at greater risk of depression and anxiety disorders at earlier ages than males, and this may partly account for their preponderance in rates of adult depression. Among possible reasons accounting for the greater risk to females and at earlier ages, psychological attributes such as neuroticism may be key determinants, acting as vulnerability factors in response to life events (Wilhelm et al, 1997).

Depressive episodes in childhood and adolescence, rather than those at older ages, predict more episodes and longer duration of depression in adult life and provide a strong link between adverse experiences in childhood and adult depression (Kendler et al, 1993; Parker et al, 1997; Bifulco et al, 1998). Considerable attention has been devoted to the role of anxiety. Using discrete-time survival models, Kessler et al (1996) showed that the associations between major depression and first onsets of other mental disorders in the same year as depression were generally strong and persistent over many years, and especially so for generalised anxiety. More recently, Parker et al (1997) assessed risk factors differentiating early-onset (that is, at an age younger than 26 years) from late-onset depression and found that anxiety disorders were more likely to precede early-onset than late-onset depression. Finally, Breslau et al (1995) showed that controlling for prior anxiety disorders reduced by more than $50 \%$ the size of the estimated association between gender and depression.

\section{Social roles and cultural norms}

The identification of individuals at high risk for developing depression, based on socio-demographic variables and data collected across different countries and cultural groups, indicates that social roles and cultural influences contribute to a female preponderance in depression rates.

Detailed reviews of epidemiological findings (Bebbington, 1996, 1998) suggest that marriage may have detrimental effects in females, possibly due to gender-specific demands posed by marriage and the resulting limited number of roles available to females. Similar reasons may explain why looking after small children is associated with greater risk of depression in females. Both home-making and child care reduce the likelihood of females being in paid employment or put additional responsibilities on those who are employed. Married females with no paid employment have to rely for identity and self-esteem on the role of housewife, a role that carries many frustrating elements and has been increasingly devalued in modern societies. On the other hand, females entering the job market face economic discrimination and job inequality along with role overload and role conflict caused by concurrent primary responsibility for household chores and child care. Although employment tends to have beneficial effects on psychological well-being, these effects may be reduced or reversed where there is role conflict and overload.

Indirect evidence for the strong effect exerted by social and cultural factors is provided by those studies showing no, or limited, gender differences in depression rates: in samples where males and females are matched for the putative social determinants of depression (Jenkins, 1985; Wilhelm et al, 1997); and in cultural groups where high value is attached to the female role, such as in Mediterranean countries (Mavreas et al, 1986; Vázquez-Barquero et al, 1987), among the Old Order Amish (Egeland \& Hostetter, 1983) or among orthodox Jews in the London area (Loewenthal et al, 1995).

The 'role strain' hypothesis is unlikely to account entirely for gender differences in depression. For example, despite significant associations between emotional distress and role underload (in both genders) or role overload (in females), in a representative sample of private households in England, Wales and Scotland, gender differences in emotional distress persisted after controlling for socio-economic status, number of social roles and occupancy of traditional female caring and domestic roles (Weich et al, 1998).

\section{Life events}

Stressful life events retain a substantial causal relationship with the onset of depressive episodes (Kendler et al, 1999). No clear evidence is available on the differential role of life events in males and females, but the quality of experience associated with life events may contribute to gender differences in depression.

The assumption that females might be at higher risk of depression owing to higher rates of adverse life events has received inconsistent support, with some studies showing gender differences in the expected direction and others finding similar levels of life events in males and females. In any case, the excess of life events in females has not been found to account entirely for their higher frequency of affective disorders (Bebbington, 1996).

Older studies simply recorded the level of life events and did not assess whether males and females differ in the meaning they attach to them. Craig (1996) reported that experiences of defeat, humiliation and entrapment were at the heart of depressive episodes in males and females, although females were far more likely to report such experiences, possibly because of their distinctive social circumstances. Similarly, Wilhelm et al (1998) found no gender differences in frequency of life events or in anticipated impact of pleasant and unpleasant events, whereas the actual impact of unpleasant events was rated higher by females.

An increased risk of the onset of depression may reasonably be expected when severe events occur in life domains to which individuals attach a strong sense of value and commitment. Individuals with few overvalued goals and/or lacking an intimate sense of perceived choice are at high risk since they are left with few alternatives for self-definition and self-evaluation when their main goals are threatened. Both of these situations are more likely for females. Consistent with a role hypothesis, a study of couples reported that the excess of depression onset among females following adverse life events was entirely restricted to crises involving children, housing or reproductive problems. This occurred among those couples with a clear gender difference in the associated roles, resulting in increased responsibility of females in these areas (Nazroo et al, 1997). These findings suggest that biological sex represents a useful starting approach in research on gender differences in depression, provided that the observed differences are then related to sociocultural roles and norms by considering an individual's multiple social identities within his or her current and biographical contexts. 


\section{Vulnerability and coping style}

The role of adverse life events in producing depression is mostly mediated through interaction with individual vulnerability, as expressed by personality characteristics, attributional style and cognitive coping (Brewin, 1996; Hänninen \& Aro, 1996). Familial aggregation of depressive episodes, temperamental features such as low self-esteem, and life events suggest that genetic factors may influence stable personal characteristics involved both in vulnerability to depression and in exposure to high-risk environments (McGuffin et al, 1988; Kendler et al, 1993, 1998).

Bebbington (1996) has reviewed the evidence on the effects produced by expectations and attributional style in depression. Expectations of negative outcomes and of helplessness lead to hopelessness, which may progress into depression. The experience is moderated by causal attributions to events, by evaluation of their consequences and by the related inferences about the self. Individuals at risk of depression have been described as characterised by globality (that is, failure is related to factors applying across a variety of situations); stability (namely, factors responsible for failure are unlikely to change over time); and internality (where the individual regards himself or herself as relatively incompetent). At present, there is insufficient support for the notion that the cognitive characteristics of females are more consistent with a depressive attributional style than those of males.

According to Nolen-Hoeksema (1987), the increased vulnerability of females to depression is mainly related to gender differences in coping with an initial lowering of mood, rather than in personality characteristics of assertiveness and passivity, which may predispose to depression. Specifically, males tend to distract themselves from their mood by engaging in physical or instrumental activities, whereas females are less active and ruminate over the possible causes and implications of their depression, thus helping to prolong the depressed mood. Indeed, less-effective coping responses involving verbal and self-consolatory strategies have been shown to occur more frequently in females (Hänninen \& Aro, 1996).

Neurocognitive studies have suggested that verbal strategies like rumination may produce increased activity of the left posterior hemisphere, whereas physical activity stimulates the right posterior hemisphere. The tendency to activate the left hemisphere as opposed to the right hemisphere under a variety of circumstances has been related to vulnerability to depression. To the extent that the right hemisphere influences the hypothalamicpituitary-adrenal axis, physical activity may be able to provide a normative restructuring of the axis, whose function is affected in depression (Heller, 1993).

\section{Social support}

Poor social support is related to onset and relapse of depression either through a direct effect unrelated to levels of concurrent adversities or through a buffering action which adds to the effect of adversities (Brugha, 1990). However, levels of social support do not seem to contribute to gender differences in depression.

It has been claimed that females have a stronger affiliative style than males, since they require greater social support for their psychological health. As a consequence, females may be more vulnerable to events affecting their close emotional ties and more likely to develop depression in response to them ('cost of caring' hypothesis). Contrary to expectation, there is no evidence for the excess of depression among females being caused by reduced social support. Research on the association between social support and depression in males and females has provided controversial findings, with some studies reporting that social support is equally important in males and females as a predictor of recovery from depression, and others showing greater beneficial effects or even a detrimental action in either of the two genders (Bebbington, 1996).

\section{Genetic factors}

Although genetic factors retain a strong influence on liability to depression, they do not seem to contribute to the increased risk to females by a direct mechanism. A recent population-based twin study of lifetime major depression showed that the degree of genetic liability to depression is similar in males and females who share most of the genetic risk factors (Kendler \& Prescott, 1999). Another study confirmed these findings for major depression defined according to DSM-IV (American Psychiatric Association, 1994) criteria, but not for other definitions of major depression (Bierut et al, 1999). On the other hand, genetic factors might indirectly increase vulnerability to depression in one gender through temperamental features associated with low self-esteem and reduced social support or social integration (Kessler et al, 1992; McGuffin et al, 1996; Kendler et al, 1998).

In the search for genetic factors implicated in gender differences in depression, attention has been paid both to genes contributing to sexual dimorphism and to genes resulting in family resemblance. No empirical evidence has supported a link between the preponderance of females in depression and dominant genes located on the X-chromosome (Faraone et al, 1987). The assumption that females have lower illness thresholds than males (threshold liability model) has received no support, since the risk of recurrence of depressive illness seems to be independent of the proband's gender (Merikangas et al, 1985). An alternative explanation may be that females have greater liability to depression owing to systematic non-familial biological and/or environmental differences, with familial transmission contributing to variation around the means (Rice et al, 1984).

\section{Gonadal hormones}

Gonadal hormones influence neurotransmitter functioning and circadian rhythms through both genomic and non-genomic effects and contribute to personality features and coping responses to stress (Parry, 1995), but other biological factors and environmental variables seem to be more prominent in depression (Seeman, 1997).

A sharp increase in depression rates in females occurs usually around mid-puberty. A direct relationship between levels of gonadal hormones and negative affect has been reported, although the social impact of puberty, the resulting cognitive changes and the rising levels of life stress also can be involved. A pathogenetic role of early pubertal timing has not been confirmed by recent studies, which showed that pubertal status was a better predictor of the emergence of depression than the timing of pubertal transition (Bebbington, 1996; Angold et al, 1998; Silberg et al, 1999). Genetic factors influencing the risk of depression and negative life events have been reported to interact with pubertal status and gender to produce higher depression rates in adolescent girls, suggesting that 
the genetic predisposition to depression and to stressful events may be 'switched on' at puberty in females (Silberg et al, 1999).

\section{Other hormonal factors}

Activation of the hypothalamic-pituitaryadrenal axis, impaired negative feedback control and associated adrenal hypertrophy are mediators (and markers) of the environmental influences on depression onset (Checkley, 1996). The axis seems to be more reactive to stress in females than in males, possibly due to a modulating role of gonadal hormones (Weiss et al, 1999). However, contrasting findings have been reported on the proposed role of the axis in producing gender differences in depression. Halbreich \& Lumley (1993) showed that age has a differential effect on the increase of cortisol plasma levels in males and females. A correlation between cortisol levels and age was detected in younger females but not in males or post-menopausal females, suggesting that hormonal changes during the menstrual cycle may contribute to an imbalance in plasma levels of cortisol. On the other hand, Young (1995) found that post-menopausal females with recurrent depression had higher postdexamethasone free cortisol than premenopausal females with depression, indicating that in pre-menopausal females oestrogens might limit the adverse sequelae of hypocortisolaemia.

Another vulnerability factor relates to the function of the hypothalamic-pituitarythyroid axis, since about $25 \%$ of depressed subjects show an abnormal increase of plasma levels of thyroid-stimulating hormone after intravenous thyrotropinreleasing hormone, and autoimmune thyroiditis and other thyroid abnormalities have been related to the onset of depression (Whybrow, 1995). Although disorders of this axis are more common in females than in males, they can hardly account for the observed gender differences in depression.

\section{Neurotransmitter systems}

Gender differences have been reported in two neurotransmitter systems traditionally implicated in the pathophysiology of depression (namely noradrenalin and serotonin), but their role is still unclear.

The changing rate of plasma levels of 3methoxy-4-hydroxyphenylglycol with age may differ in males and females with depression. Whereas most females with depression are below or above the reference range according to age, most males with depression lie within reference ranges. By inference, an age-related gender difference in vulnerability to dysregulation of the noradrenalin system has been suggested (Halbreich \& Lumley, 1993). Similarly, the ageing process of some serotonin systems might be more apparent in females than males, as measured by diurnal variations in imipramine binding and serotonin uptake in platelets. The likelihood of a relationship between food intake, weight gain and depressed mood in females, with brain serotonin being involved in these disturbances of mood and appetite, is intriguing (Wurtman, 1993).

\section{Gender differences in depression}

Although artefactual determinants may enhance a female preponderance in depressive disorders to some extent, gender differences in depression are genuine. Determinants of such differences are far from being established and their combination into integrated aetio-pathogenetic models continues to be lacking. At present, adverse experiences in childhood, depression and anxiety disorders in childhood and adolescence, sociocultural roles with related adverse experiences, and psychological attributes related to vulnerability to adverse life events and coping skills are likely to be involved. Genetic and biological factors and poor social support, however, have few or no effects in the emergence of gender differences.

\section{REFERENCES}

American Psychiatric Association (1994) Diagnostic and Statistical Manual of Mental Disorders (4th edn) (DSM-IV). Washington, DC: APA.

Angold, A., Costello, E. J. \& Worthman, C. M. (1998) Puberty and depression: the roles of age, pubertal status and pubertal timing. Psychological Medicine, 28 $51-6 \mid$.

Angst, J. \& Dobler-Mikola, A. (1984) Do the diagnostic criteria determine the sex ratio in depression? Journal of Affective Disorders, 7, 189-198.

Bebbington, P. E. (1996) The origins of sex differences in depressive disorder: bridging the gap. International Review of Psychiatry, 8, 295-332.

- (1998) Sex and depression. Psychological Medicine, 28, $1-8$.

Bierut, L. J., Heath, A. C., Bucholz, K. K., et al (1999) Major depression disorder in a community-based twin sample. Archives of General Psychiatry, 56, 557-563.

Bifulco, A., Brown, G.W., Moran, P., et al (1998) Predicting depression in women: the role of past and present vulnerability. Psychological Medicine, 28, 39-50.
Bracke, P. (1998) Sex differences in the course of depression: evidence from a longitudinal study of a representative sample of the Belgian population. Socia Psychiatry and Psychiatric Epidemiology, 33, 420-429.

Breslau, N., Schultz, L. \& Peterson, E. (1995) Sex differences in depression: a role for preexisting anxiety Psychiatry Research, 58, 1-12.

Brewin, C. R. (1996) Cognitive processing of adverse experiences. In The Causes of Depression (eds A. H. Mann \& M. J. Owen). International Review of Psychiatry, 8 333-339.

Bromet, E. J., Dunn, L. O., Conell, M. M., et al (1986) Long-term reliability of diagnosing lifetime major depression in a community sample. Archives of General Psychiatry, 43, 435-440.

Brown, G.W. (1996) Genetics of depression: a social science perspective. International Review of Psychiatry, $\mathbf{8}$, 387-402.

Brugha, T. S. (1990) Social networks and support. Current Opinion in Psychiatry, 3, 264-268.

Cadoret, R. J., Winokur, G., Langbehn, D., et al (1996) Depression spectrum disease, I: The role of geneenvironment interaction. American Journal of Psychiatry, I53, 892-899.

Cantwell, D. P., Lewinsohn, P. M., Rohde, P., et al (1997) Correspondence between adolescent report and parent report of psychiatric diagnostic data. Journal of the American Academy of Child and Adolescent Psychiatry, 36, 610-619.

Checkley, S. (1996) The neuroendocrinology of depression. International Review of Psychiatry, 8 , 373-378.

Coryell, W., Endicott, J. \& Keller, M. (1992) Major depression in a nonclinical sample: demographic and clinical risk factors for first onset. Archives of General Psychiatry, 49, II7-125.

Craig, T. K. J. (1996) Adversity and depression International Review of Psychiatry, 8, 34I-353.

Dawson, D. A. \& Grant, B. F. (1998) Family history of alcoholism and gender: their combined effects on DSMIV alcohol dependence and major depression. Journal of Studies on Alcohol, 59, 97-106.

Dohrenwend, B. P. (1989) The problem of validity in field studies of psychological disorders revisited. In The Validity of Psychiatric Diagnosis (eds L. N. Robins \& J. E. Barrett), pp. 35-53. New York: Raven Press.

Egeland, J. A. \& Hostetter, A. M. (1983) Amish Study, I: Affective disorders among the Amish, 1976-1980. American Journal of Psychiatry, 140, 56-61.

Faraone, S.V., Lyons, M. J. \& Tsuang, M.T. (1987) Sex differences in affective disorder: genetic transmission. Genetic Epidemiology, 4, 33I-343.

Farmer, A. E. (1996) The genetics of depressive disorders. International Review of Psychiatry, 8 369-372.

Fennig, S., Schwartz, J. E. \& Bromet, E. J. (1994) Are diagnostic criteria, time of episode and occupational impairment important determinants of the female:male ratio for major depression? Journal of Affective Disorders, 30, 147-154.

Frank, E., Carpenter, L. L. \& Kupfer, D. J. (1988) Sex differences in recurrent depression: are there any that are significant? American Journal of Psychiatry, 145, $4 \mid-45$.

Gijsbers van Wijk, C. M., Huisman, H. \& Kolk, A. M. (1999) Gender differences in physical symptoms and illness behavior: a health diary study. Social Science and Medicine, 49, 1061-1074. 
Halbreich, U. \& Lumley, L. A. (1993) The multiple interactional biological processes that might lead to depression and gender differences in its appearance. Journal of Affective Disorders, 29, 159-173.

Hänninen, V. \& Aro, H. (1996) Sex differences in coping and depression among young adults. Social Science and Medicine, 43, 1453-1460.

Harrington, R. (1996) Family-genetic findings in child and adolescent depressive disorders. International Review of Psychiatry, 8, 355-368.

Heller, W. (1993) Gender differences in depression: perspectives from neuropsychology. Journal of Affective Disorders, 29, 129-143.

Jenkins, R. (1985) Sex differences in minor psychiatric morbidity. Psychological Medicine Monograph Supplement, 7, I-53.

Kendler, K. S. \& Prescott, C. A. (1999) A populationbased twin study of lifetime major depression in men and women. Archives of General Psychiatry, 56, 39-44.

\section{_, Kessler, R. C., Neale, M. C., et al (1993) The} prediction of major depression in women: toward an integrated etiologic model. American Journal of Psychiatry, I50, |139-||48.

_ Walters, E. E., Neale, M. C., et al (1995) The structure of the genetic and environmental risk factors for six major psychiatric disorders in women. Archives of General Psychiatry, 52, 374-383.

_ , Davis, C. G. \& Kessler, R. C. (1997) The familial aggregation of common psychiatric and substance use disorders in the National Comorbidity Survey: a family history study. British Journal of Psychiatry, 170, 541-548.

\section{_, Gardner, C. O. \& Prescott, C. A. (1998) A}

population-based twin study of self-esteem and gender. Psychological Medicine, 28, 1403-1409.

_ , Karkowski, L. M. \& Prescott, C. A. (1999) Causal relationship between stressful life events and the onset of major depression. American Journal of Psychiatry, I56 837-841.

Kessler, R. C., Kendler, K. S., Heath, A., et al (1992) Social support, depressed mood, and adjustment to stress: a genetic epidemiologic investigation. Journal of Personality and Social Psychology, 62, 257-272.

__, McGonagle, K. A., Swartz, M., et al (1993) Sex and depression in the National Comorbidity Survey I: Lifetime prevalence, chronicity and recurrence. Journal of Affective Disorders, 29, 85-96.

\section{—, Nelson, C. B., McGonagle, K. A., et al (1996)} Comorbidity of DSM-III-R major depressive disorder in the general population: results from the US National Comorbidity Survey. British Journal of Psychiatry, 168 (suppl. 30), 17-30.

Loewenthal, K., Goldblatt, V., Gorton, T., et al (1995) Gender and depression in Anglo-Jewry. Psychological Medicine, 25, 105I-1064.

Lyness, J. M., Cox, C., Curry, J., et al (1995) Older age and the underreporting of depressive symptoms. Journal of the American Geriatric Society, 43, 216-22I.

Mavreas, V. G., Beis, A., Mouyias, A., et al (1986) Prevalence of psychiatric disorders in Athens: a community study. Social Psychiatry, 21, 172-181.

McGuffin, P., Katz, R., Bebbington, P. (1988) The Camberwell Collaborative Depression Study. III. Depression and adversity in the relatives of depressed probands. British Journal of Psychiatry, 152, 775-782.

_ , _ , Watkins, S., et al (1996) A hospital-based twin register of the heritability of DSM-IV unipolar depression. Archives of General Psychiatry, 53, 129-136.

\section{CLINICAL IMPLICATIONS}

Biological sex is an immutable socio-demographic variable not influenced by disease and thus is a useful starting point in the investigation of risk factors for depression.

- Investigations into gender differences in depression can assess the relative importance of risk factors from different domains, including biological, psychological and sociocultural influences.

- Clinically important risk factors for predominance of females in depression are: sexual abuse and adverse childhood experiences; role limitation with associated lack of choice, role overload and competing social roles; psychological attributes related to vulnerability to life events and coping skills.

\section{LIMITATIONS}

- Few efforts have been made to combine putative risk factors for gender differences in depression into integrated aetio-pathogenetic models because of difficulties arising in empirical testing.

- The approach based on biological sex is rarely combined with the study of developmental processes underlying the acquisition of gender identity.

- There is a relative lack of longitudinal studies testing several variables simultaneously for their ability to predict the appearance of depressive episodes and related gender differences.

MARCO PICCINELLI, PhD, Unità Operativa di Psichiatria I, Azienda Ospedaliera Ospedale di Circolo e Fondazione Macchi, Varese, Italy; GREG WILKINSON, FRCPsych, Academic Department of Psychiatry, Royal Liverpool University Hospital, Liverpool, UK

Correspondence: Dr Marco Piccinelli, Servizio Psichiatrico di Diagnosi e Cura, Presidio Ospedaliero del Verbano - sede di Cittiglio, via Marconi 40, 21033 Cittiglio (Varese), Italy. Tel: +39 0332607 245; fax: +39 033262669

Merikangas, K. R., Weissman, M. M. \& Pauls, D. L. (1985) Genetic factors in the sex ratio of major depression. Psychological Medicine, I5, 63-69.

Nazroo, J. Y., Edwards, A. C. \& Brown, G. W. (1997) Gender differences in the onset of depression following a shared life event: a study of couples. Psychological Medicine, 27, 9-19.

Nolen-Hoeksema, S. (1987) Sex differences in unipolar depression: evidence and theory. Psychological Bulletin, I0I, 259-282.

Parker, G., Wilhelm, K. \& Asghari, A. (1997) Early onset depression: the relevance of anxiety. Social Psychiatry and Psychiatric Epidemiology, 32, 30-37.

Parry, B. L. (1995) Sex hormones, circadian rhythms and depressive vulnerability. Depression, 3, 43-48.

Piccinelli, M. \& Gomez Homen, F. (1997) Gender Differences in the Epidemiology of Affective Disorders and Schizophrenia, pp. 3-59. Geneva: World Health Organization.

Rice, J., Reich, T., Andreasen, N. C., et al (1984) Sexrelated differences in depression: familial evidence. Journal of Affective Disorders, 7, 199-210.
Rodgers, B. (1994) Pathways between parental divorce and adult depression. Journal of Child Psychology and Psychiatry, 35, 1289-1308.

Seeman, M. V. (1997) Psychopathology in women and men: focus on female hormones. American journal of Psychiatry, 154, 1641-1647.

Silberg, J., Pickles, A., Rutter, M., et al (1999) The influence of genetic factors and life stress on depression among adolescent girls. Archives of General Psychiatry, 56, 225-232.

Silverstein, B. (1999) Gender differences in the prevalence of clinical depression: the role played by depression associated with somatic symptoms. American journal of Psychiatry, I56, 480-482.

Spinhoven, P. \& Kooiman, C. G. (1997) Defense style in depressed and anxious psychiatric outpatients: an explorative study. Journal of Nervous and Mental Disease, 185, 87-94.

Stallings, M. C., Cherny, S. S., Young, S. E., et a (1997) The familial aggregation of depressive symptoms, antisocial behavior, and alcohol abuse. American Journal of Medical Genetics, 74, I83-191. 
Steer, R. A., Beck, A. T. \& Brown, G. (1989) Sex differences on the revised Beck Depression Inventory for outpatients with affective disorders. Journal of Personality Assessment, 53, 693-702.

Stefànsson, J. G., Lindal, E., Bjornsson, J. K., et a (1994) Period prevalence rates of specific mental disorders in an Icelandic cohort. Social Psychiatry and Psychiatric Epidemiology, 29, 119-125.

Stommel, M., Given, G. A., Given, C. W., et al (1993) Gender bias in the measurement properties of the Centre for Epidemiologic Studies Depression Scale (CES-D). Psychiatry Research, 49, 239-250.

Tousignant, M., Brosseau, R. \& Tremblay, L. (1987) Sex biases in mental health scales: do women tend to report less serious symptoms and confide more than men? Psychological Medicine, 17, 203-215.

Vázquez-Barquero, J. L., Diez-Manrique, J. F., Pena, C., et al (1987) A community mental health survey in Cantabria: a general description of morbidity. Psychological Medicine, 17, 227-242.
Veijola, J., Puukka, P., Letinen, V., et al (1998) Sex differences in the association between childhood experiences and adult depression. Psychological Medicine, 28, $21-27$.

Weich, S., Sloggett, A. \& Lewis, G. (1998) Social roles and gender difference in the prevalence of common mental disorders. British Journal of Psychiatry, 173 489-493.

Weiss, E. L., Longhurst, J. G. \& Mazure, C. M. (1999) Childhood sexual abuse as a risk factor for depression in women: psychosocial and neurobiological correlates. American Journal of Psychiatry, I56, 816-828.

Weissman, M. M. \& Klerman, G. L. (1977) Sex differences and the epidemiology of depression. Archives of General Psychiatry, 34, 98-III.

Whybrow, P. C. (1995) Sex differences in thyroid axis function: relevance to affective disorder and its treatment. Depression, 3, 33-42.
Wilhelm, K. \& Parker, G. (1994) Sex differences in lifetime depression rates: fact or artefact? Psychologica Medicine, 24, 97-III.

—, Parker, G. \& Hadzi-Pavlovic, D. (1997) Fifteen years on: evolving ideas in researching sex differences in depression. Psychological Medicine, 27, 875-883.

_, _ \& Dewhurst, J. (1998) Examining sex differences in the impact of anticipated and actual life events. Journal of Affective Disorders, 48, 37-45.

Wurtman, J. J. (1993) Depression and weight gain: the serotonin connection. Journal of Affective Disorders, 29 183-192.

Young, E. A. (1995) Glucocorticoid cascade hypothesis revisited. Role of gonadal steroids. Depression, 3, 20-27.

Young, M. A., Scheftner, W. A., Fawcett, J., et al (1990) Gender differences in the clinical features of unipolar major depressive disorder. Journal of Nervous and Mental Disease, 178, 200-203. 